\title{
Hematopoietic stem and progenitor cells take the route through the bone marrow endothelium
}

\author{
Lydia Kalafati ${ }^{1,2}$ and Triantafyllos Chavakis ${ }^{1}$
}

\begin{abstract}
'Institute for Clinical Chemistry and Laboratory Medicine, University Hospital and Faculty of Medicine, Technische Universität Dresden, Dresden and ${ }^{2}$ National Center for Tumor Diseases, Partner Site Dresden, Dresden and German Cancer Research Center Heidelberg, Heidelberg, Germany
\end{abstract}

E-mail: TRIANTAFYLLOS CHAVAKIS - triantafyllos.chavakis@uniklinikum-dresden.de

doi:10.3324/haematol.2020.262113

$\mathrm{H}$ ematopoietic stem cell transplantation (HSCT) is an established therapeutic approach for various hematologic diseases, particularly hematologic malignancies. A prerequisite for successful HSCT is the recruitment of infused hematopoietic stem and progenitor cells (HSPC) to the bone marrow (BM). A critical component in this process is the transmigration of HSPC through the BM endothelium. But how do HSPC actually cross the BM endothelium? Given that HSCT has been performed in the clinics for decades now, surprisingly little is known about the mechanisms underlying the transmigration of HSPC through the BM endothelium. A study by Rademakers et al. ${ }^{1}$ in this issue of Haematologica provides major novel insights into this important question. In particular, the authors show that HSPC engage preferentially in the transcellular route to migrate through the BM endothelium by using podosome-like structures.

Hematopoiesis, i.e., the generation of blood cells, takes place in the BM. Hematopoietic stem cells (HSC) lie at the top of the hematopoietic hierarchy and are characterized by the capacity of self-renewal and multi-lineage differentiation giving rise to all mature blood cells. ${ }^{2}$ HSPC play also an important role in demand-adapted hematopoiesis, as they respond rapidly to inflammatory or infectious challenges by proliferative expan- sion and enhanced differentiation to myeloid cells. ${ }^{3}$ The function of HSPC in the BM is supported by the special environment of the hematopoietic niche that consists of the extracellular matrix and several cells, such as endothelial cells and mesenchymal stromal cells. ${ }^{4}$ The niche facilitates HSPC functions, including their maintenance and their differentiation via different mechanisms. ${ }^{4}$ While vascular endothelial cells in the BM contribute to the HSC niche, they also represent the main barrier that HSC need to cross in order to arrive in the BM and repopulate the BM niche in the context of HSCT, which is an established treatment for several hematologic disorders, especially hematologic malignancies. ${ }^{5,6} \mathrm{HSC}$ engraftment in the BM in HSCT is regulated by various interactions with different components of the $\mathrm{BM}$ niche, ${ }^{4}$ however, less is known regarding the mechanisms that facilitate the initial BM homing of HSC upon transplantation.

The BM vascular endothelium expresses various adhesion molecules that facilitate adhesion of circulating cells. ${ }^{7}$ As the shear stress in sinusoidal vessels is significantly lower than in arterioles, BM sinusoids are a preferential site for HSPC homing. ${ }^{8}$ The adhesive interactions of HSPC with the BM endothelium share major similarities with the leukocyte adhesion cascade, which mediates leukocyte recruitment in inflammation. ${ }^{9}$



Figure 1 Hematopoietic stem and progenitor cells bone marror homing in the context of hematopoietic stem cell transplantation. Upon hematopoietic stem cell transplantation (HSCT), the hematopoietic stem and progenitor cells (HSPC) extravasate through the bone marrow (BM) vascular endothelium enter and repopulate the BM niche. The $\mathrm{BM}$ endothelium expresses several adhesion molecules, such as selectins and VCAM-1, which facilitate rolling and firm adhesion of HSPC on the endothelial cells. Subsequently, the HSPC preferentially migrate through the endothelial cell body, designated as transcellular transmigration. VE-cadherin regulates vascular endothelial integrity by stabilizing the endothelial cell-cell junctions. Following inhibition of VE-cadherin, vascular permeability increases and HSPC may also migrate through the endothelial cell junctions in a paracellular manner. 
Specifically, the rolling of HSPC and their subsequent firm adhesion onto the vascular endothelial cells of the BM are mediated by endothelium-expressed selectins and vascular cell adhesion molecule-1 (VCAM-1), respectively. ${ }^{10,11}$ VCAM-1 on the BM endothelium interacts primarily with integrin $\alpha 4 \beta 1$, also named very late antigen-4 (VLA-4) on HSPC. ${ }^{11}$ Furthermore, the interaction between integrin $\alpha 4 \beta 7$ on HSPC and mucosal addressin cell adhesion molecule 1 (MAdCAM1) on the BM endothelium facilitates HSPC recruitment in the context of transplantation. ${ }^{12}$

After their adhesion on the endothelium, HSPC extravasate into the $\mathrm{BM}$ niche. In the leukocyte adhesion cascade, immune cells may take two distinct routes to migrate across the endothelial barrier. The paracellular route is through the cell-cell junctions, while the transcellular route occurs through the endothelial cell body, with paracellular transendothelial migration being the predominant pathway at least for innate immune cells. ${ }^{13}$ However, lymphocytes may preferentially engage in the transcellular transmigration route. ${ }^{14}$ The choice between transcellular and paracellular transmigration may be context-dependent; different immune cells may have a preference for one route over the other, however, this choice may also depend on the type of endothelium in different vascular beds and tissues. ${ }^{15}$

The major regulator of endothelial cell-cell junctions and vascular integrity is vascular endothelial (VE)-cadherin; ${ }^{16}$ VE-cadherin is, therefore also a modulator of the paracellular transmigration route. Antibodies blocking VE-cadherin can promote increased vessel permeability and enhanced leukocyte diapedesis. ${ }^{17,18}$ The exact role of VE-cadherin in HSPC transendothelial migration, however remains elusive. An older in vitro study by van Buul et al. ${ }^{19}$ showed that inhibition of VE-cadherin in human BM endothelial cells not only increased their permeability but also promoted the transendothelial migration of human $\mathrm{CD} 34^{+}$hematopoietic progenitor cells. ${ }^{19}$ The same group followed up on this previous finding in their article by Rademakers and collegues, ${ }^{1}$ published in this issue of Haematologica. In this article, the authors provide important novel insights into the role of VE-cadherin for the vascular integrity in the BM and on the mechanisms that HSPC employ to cross the BM endothelial barrier. They show that VE-cadherin is a major regulator of vascular endothelial permeability in the BM. ${ }^{1}$ Antibodymediated inhibition of VE-cadherin led to increased permeability in both sinusoids and arterioles in the BM under steady-state conditions and upon low-dose irraditation. Conversely, VE-cadherin- $\alpha$-catenin chimera knock-in animals, in which endothelial junctions are stabilized by the expression of the VE-cadherin-alpha-catenin fusion protein ${ }^{20}$ displayed reduced BM vascular permeability under normal conditions and upon irradiation. Additionally, BM homing of HSPC transferred to low-dose irradiated mice was enhanced in the presence of blocking antibody against VE-cadherin. ${ }^{1}$ Surprisingly, homing of HSPC to the $\mathrm{BM}$ was not altered in VE-cadherin- $\alpha$-catenin chimera knock-in mice. This finding could be attributed to the fact that HSPC engage preferentially the transcellular route to cross the BM endothelial barrier. Hence, transendothelial migration of HSPC is regulated in a different way than vascular permeability in the BM and only the latter is strictly dependent on VE-cadherin. ${ }^{1}$ Further mechanistic experi- ments suggested that podosome structures on HSPC mediate their transendothelial migration. Despite the enhanced HSPC recruitment to the BM upon antibody-mediated blockade of VE-cadherin long-term engraftment of HSPC in the BM was not improved. Hence, blocking VE-cadherin does not necessarily promote hematopoietic regeneration in the context of transplantation.

Taken together, this article provides an important novel insight into the mechanisms underlying HSPC homing to the $\mathrm{BM}$ in the context of transplantation. The findings may be of importance with regards to developing strategies to enhance HSPC homing and increase the efficacy of therapeutic HSC transplantation.

\section{References}

1. Rademakers T, Goedhart M, Hoogenboezem M, et al, Hematopoietic stem and progenitor cells use podosomes to transcellularly cross the bone marrow endothelium. Haematologica. 105(12):2746-2756.

2. Jacobsen SEW, Nerlov C. Haematopoiesis in the era of advanced single-cell technologies. Nat Cell Biol, 2019;21(1):2-8.

3. Chavakis T, Mitroulis I, Hajishengallis G. Hematopoietic progenitor cells as integrative hubs for adaptation to and fine-tuning of inflammation. Nat Immunol. 2019;20(7):802-811.

4. Wei Q, Frenette PS. Niches for hematopoietic stem cells and their progeny. Immunity. 2018;48(4):632-648.

5. To LB, Haylock DN, Simmons PJ, Juttner CA. The biology and clinical uses of blood stem cells. Blood. 1997;89(7):2233-2258.

6. Copelan EA. Hematopoietic stem-cell transplantation. N Engl J Med. 2006;354(17):1813-1826.

7. Lapidot T, Dar A, Kollet O. How do stem cells find their way home? Blood. 2005;106(6):1901-1910.

8. Perlin JR, Sporrij A, Zon LI. Blood on the tracks: hematopoietic stem cell-endothelial cell interactions in homing and engraftment. J Mol Med (Berl). 2017;95(8):809-819.

9. Nourshargh S, Alon R. Leukocyte migration into inflamed tissues. Immunity. 2014;41(5):694-707.

10. Frenette PS, Subbarao S, Mazo IB, von Andrian UH, Wagner DD. Endothelial selectins and vascular cell adhesion molecule-1 promote hematopoietic progenitor homing to bone marrow. Proc Natl Acad Sci U S A. 1998;95(24):14423-14428.

11. Papayannopoulou T, Craddock C, Nakamoto B, Priestley GV, Wolf NS. The VLA4/VCAM-1 adhesion pathway defines contrasting mechanisms of lodgement of transplanted murine hemopoietic progenitors between bone marrow and spleen. Proc Natl Acad Sci U S A. 1995;92 (21):9647-9651.

12. Katayama, Y, Hidalgo A, Peired A, Frenette PS. Integrin alpha4beta7 and its counterreceptor MAdCAM-1 contribute to hematopoietic progenitor recruitment into bone marrow following transplantation. Blood. 2004,104(7):2020-6.

13. Vestweber D, Wessel F, Nottebaum AF. Similarities and differences in the regulation of leukocyte extravasation and vascular permeability. Semin Immunopathol. 2014;36(2):177-192.

14. Carman CV. Mechanisms for transcellular diapedesis: probing and pathfinding by 'invadosome-like protrusions'. J Cell Sci. 2009;122(Pt 17):3025-3035.

15. Martinelli R, Zeiger AS, Whitfield M, et al. Probing the biomechanical contribution of the endothelium to lymphocyte migration: diapedesis by the path of least resistance. J Cell Sci. 2014;127(Pt 17):3720-3734.

16. Lagendijk AK, Hogan BM. VE-cadherin in vascular development: a coordinator of cell signaling and tissue morphogenesis. Curr Top Dev Biol. 2015;112:325-352.

17. Corada M, Mariotti M, Thurston G, et al. Vascular endothelial-cadherin is an important determinant of microvascular integrity in vivo. Proc Natl Acad Sci U S A. 1999;96(17):9815-9820.

18. Gotsch U, Borges E, Bosse R, et al. VE-cadherin antibody accelerates neutrophil recruitment in vivo. J Cell Sci. 1997;110( Pt 5):583-588.

19. van Buul JD, Voermans C, van den Berg V, et al. Migration of human hematopoietic progenitor cells across bone marrow endothelium is regulated by vascular endothelial cadherin. J Immunol. 2002;168(2):588-596.

20. Schulte D, Küppers V, Dartsch N, et al. Stabilizing the VE-cadherincatenin complex blocks leukocyte extravasation and vascular permeability. EMBO J. 2011;30(20):4157-4170. 